\title{
Un método para el análisis de frecuencia regional de lluvias máximas diarias: aplicación en los Andes bolivianos
}

\author{
A method for regional frequency analysis of maximum daily rainfall: application in \\ the Bolivian Andes
}

\author{
José Antonio Luna Vera ${ }^{1} \quad$ Ramón Domínguez Mora² \\ Recibido 9 de abril de 2012, aceptado 14 de enero de 2013 \\ Received: April 9, 2012 Accepted: January 14, 2013
}

\begin{abstract}
RESUMEN
Se presenta un análisis de frecuencia regional con series de lluvia diaria máxima anual para una zona con escasa información. La compleja orografía de montañas y el altiplano de una región en la cordillera de Los Andes, Bolivia, produce diferentes patrones de lluvia diaria. La combinación de los Momentos-L y el análisis de conglomerados resultan adecuados para identificarlas regiones homogéneas de las series máximas anuales. El trabajo desarrollado define 4 regiones homogéneas. La región 1 comprende las estaciones ubicadas en el altiplano y la zona Sur Este. La región 2 abarca el altiplano central y la cuenca del Río La Paz, compuesto por cuencas interandinas. La 3 delimita claramente las estaciones de la zona tropical amazónica; y la 4 está compuesta por estaciones ubicadas en las montañas del Norte. Se probaron diversas distribuciones para el análisis regional de frecuencias aplicando la técnica de estaciones-año; los mejores resultados se obtuvieron con las funciones Gumbel y Doble Gumbel. Finalmente se expresan las ecuaciones regionales y se comparan con algunas series puntuales de cada región, con el objeto de verificar la aplicabilidad de la metodología propuesta para fines de diseño hidrológico.
\end{abstract}

Palabras clave: Regionalización, lluvia diaria máxima, momentos-L, método estaciones-año, método k-medias, homogeneización, análisis de frecuencia.

\begin{abstract}
A regional frequency analysis of daily annual maximum rainfall series for an area with poor information is presented. The complex topography mountains and the highlands region in the Cordillera de Los Andes, Bolivia, produce different patterns of daily rainfall. The combination of L-Moments and cluster analysis are adequate to identify the homogeneous regions of the annual maximum series. The work defines 4 homogeneous regions. Region 1 includes the stations located in the highlands and south-east. Region 2 covers the central highlands and La Paz River Basin, consisting of inter-Andean basins. Region 3 clearly defines the Amazonian basin stations and 4 is composed of stations located in the northern mountains. Different distributions were tested for regional frequency analysis using the Index Flood method, the best results were obtained with the Double Gumbel and Gumbel functions. Finally, regional equations are compared with some specified series of each region, in order to verify the applicability of the proposed methodology for hydrological design purposes.
\end{abstract}

Keywords: Regionalization, maximum daily rainfall, L-moments, index-flood method, $k$-means method, homogenization, frequency analysis.

1 Facultad de Ingeniería. División de Estudios de Posgrado de la Facultad de Ingeniería. Universidad Nacional Autónoma de México. Ciudad Universitaria, CP 04510, Coyoacán, México, DF. E-mail: JLunaV @iingen.unam.mx

2 Instituto de Ingeniería. Universidad Nacional Autónoma de México. Cd. Universitaria. CP 04510. México, DF. E-mail: RDominguezM@iingen.unam.mx 


\section{INTRODUCCIÓN}

La estimación de crecidas en cuencas de montañas de Los Andes, Sud América, se basa tradicionalmente en criterios empíricos y otros métodos paramétricos como la Fórmula Racional, el Hidrograma Unitario Sintético y el método del Soil Conservation Service [1], como lo describe Chow [2]. También es común aplicar el modelo hidrológico del U.S. Army Corp Engineers, HEC-HMS[3], basado en la lluvia diaria y una distribución de la lluvia en el tiempo para duraciones de 6 ó 24 horas [1], o bien con una discretización de lluvia fundamentada en las tormentas puntuales medidas en estaciones pluviográficas locales [4-5].

La zona de estudio tiene una red meteorológica poca densa y no existen estudios regionales que coadyuven a mejorar la estimación de los eventos extremos. En las cuencas no aforadas se asumen lluvias de estaciones vecinas y ésta decisión puede llevar a errores de diseño, ya sea sub-dimensionando o sobredimensionando las obras hidráulicas. Las ventajas de un enfoque regional para el análisis de lluvias máximas se traducen en una mejora de estimaciones de las tormentas de diseño, así como la capacidad para estimar avenidas de diseño en sitios sin datos o con escasa información.

La zona de estudio presenta distintos patrones de precipitación, como lluvias frontales de invierno (Frente frío polar del sur) y una combinación de factores orográficos y convectivos locales muy intensos, característicos del verano. Las tormentas del primer tipo son de menor frecuencia, pero suelen tener magnitudes importantes en tiempos prolongados. En cambio, las otras tormentas son de mayor impacto e importancia, debido a las grandes magnitudes de lluvia concentrada en intervalos de tiempo cortos. Se espera que un enfoque regional de las precipitaciones extremas permita explicar los distintos tipos de lluvias, logrando una mejora en la predicción de eventos extremos.

Se han desarrollado recientes avances en el análisis de frecuencias que incluyen el uso de Momentos- $L$ [6-10]. Su validez y aplicabilidad a la hidrología ha sido probada por diversos autores [11, 8-10, 12]. Por otra parte, el método de las Estaciones-Año [13], fue utilizado en principio para la estimación regional de caudales, fundamentado en el análisis de frecuencia con la función de distribución de probabilidad GVE Tipo I, Gumbel. En la actualidad, este método ha mostrado su aplicabilidad en el proceso de regionalización de lluvias extremas [12,14-15], y el criterio ha sido extendido para aplicarlo con otras distribuciones.

La homogeneidad de variables hidrológicas ha sido estudiada ampliamente en procesos asociados a una variable aleatoria. Actualmente los criterios para conformar grupos homogéneos son amplios; permiten incluir múltiples variables e interpretar la importancia relativa de cada una. En el artículo se emplea el método de Fisher [16] y la técnica $k$-Medias [17-18] para determinar grupos con características semejantes.

\section{REGIONALIZACIÓN}

Un problema común en la evaluación de eventos extremos de lluvias o crecidas es su estimación en sitios sin datos o con poca información; este inconveniente se corrige mediante un análisis de datos de varias estaciones vecinas. Al conformar grupos de estaciones se aprovecha la información de la región cuando ésta es homogénea. Al aplicar el concepto de homogeneidad en el análisis regional con un tipo de datos, se benefician las estimaciones, obteniéndose mayor confiabilidad de resultados, en comparación con la forma tradicional que sólo utiliza información de un sitio.

Por consiguiente, la cuantificación de eventos hidrológicos o meteorológicos en regiones con poca o nula información, se debe lograr con base a la información disponible en toda la región, hidrológica o meteorológicamente homogénea.

El análisis regional hidrológico proporciona una forma de inferir eventos hidrológicos en una zona con baja densidad de estaciones o que cuente con escasa información, a partir de las características fisiográficas y meteorológicas, así como de otras variables que determinen el comportamiento hidrológico de la misma.

Los criterios de regionalización empleados tradicionalmente tienen como finalidad lograr una delimitación territorial, basados simplemente en características geográficas, o estadísticamente sustentados en los promedios de la variable analizada. 
Existen diversos métodos para aplicar un análisis regional, pero, antes de mencionarlos, es importante indicar que su uso práctico implica dos etapas: i) Delimitación de Regiones Homogéneas y ii) Estimación Regional [10].

Delimitar regiones hidrológicas o meteorológicas homogéneas es una tarea muy compleja, puesto que implica hacer un análisis estadístico profundo de los datos observados en una región. Existen varias técnicas para formar regiones homogéneas; ver por ejemplo, Cunnane [19] Escalante y Reyes [10] y Campos [12]. Lamentablemente es frecuente que los métodos dan resultados distintos, incluso partiendo de los mismos datos. La selección de un método puede resultar contraproducente, debido a la ambigüedad del resultado obtenido. En cambio, Escalante y Reyes [10] sugieren emplear dos o más criterios, éstos pueden complementarse para obtener un resultado favorable.

\section{Momentos - L}

Hosking y Wallis [7-8] definen parámetros estadísticos de interpretación análoga a los momentos tradicionales, a los que ellos llaman Momentos-L. Estos se deducen a partir de combinaciones lineales de los momentos de probabilidad ponderada (MPP) [20], que posteriormente se convirtieron en la base del trabajo de Hosking y Wallis [8]. Estos autores afirman que un modelo se hace robusto cuando se aprovecha la información de la región. Así, el método que proponen agrupa series máximas anuales (SMA) de las estaciones de una región homogénea. La hipótesis de partida es que los datos provienen de una población base.

Los Momentos- $L$ se interpretan como un sistema alternativo para estimar los parámetros de la distribución de probabilidad. El momento de probabilidad ponderada de orden $r, \beta_{r}$, está dado por la siguiente expresión:

$$
\beta_{r}=\int_{-\infty}^{+\infty} x[F(x)]^{r} f(x) d x
$$

Donde $F(x)$ y $f(x)$ son las funciones de distribución acumulada y la función de densidad de probabilidad de $x$, respectivamente. Los momentos, $\lambda_{r}$, son combinaciones lineales de los MPP [20], $\beta_{r}$.

La regionalización que sugieren Hosking y Wallis [8] inicia con el cálculo de los estimadores de Momentos $-L, \lambda_{r}$, a través del siguiente procedimiento:

1. Se ordenan las SMA $x_{i}$ de cada estación, $j$, en forma descendente.

2. Se calculan los estimadores $\hat{\beta}$, aplicando las ecuaciones(2) a (5)

$$
\begin{gathered}
\hat{\beta} 0=\frac{1}{n} \sum_{i=1}^{n} x_{i} \\
\hat{\beta}_{1}=\frac{\sum_{i=1}^{n-1}(n-i) x_{i}}{n(n-1)} \\
\hat{\beta}_{2}=\frac{\sum_{i=1}^{n-2}(n-i)(n-i-1)(n-i-2) x_{i}}{n(n-1)(n-2)} \\
\hat{\beta}_{3}=\frac{\sum_{i=1}^{n-3}(n-i)(n-i-1)(n-i-2) x_{i}}{n(n-1)(n-2)(n-3)}
\end{gathered}
$$

Donde: $x_{i}$, valor de la serie máxima anual; $i$, orden de cada dato arreglado de mayor a menor; $n$, número de datos de la serie en cuestión.

3. Se obtienen los Momentos-L

$$
\begin{gathered}
\lambda_{1}=\hat{\beta}_{0} ; \\
\lambda_{2}=2 \hat{\beta}_{1}-\hat{\beta}_{0} ; \\
\lambda_{3}=6 \hat{\beta}_{2}-6 \hat{\beta}_{1}+\hat{\beta}_{0} ; \\
\lambda_{4}=20 \hat{\beta}_{3}-30 \hat{\beta}_{2}+12 \hat{\beta}_{1}-\hat{\beta}_{0}
\end{gathered}
$$

4. Se calculan los Momentos-L adimensionales: $\tau_{2}, \tau_{3}$ y $\tau_{4}:$

$$
\begin{aligned}
& \tau_{2}=\lambda_{2} / \lambda_{1} \\
& \tau_{3}=\lambda_{3} / \lambda_{2} \\
& \tau_{4}=\lambda_{4} / \lambda_{2}
\end{aligned}
$$

Donde $\tau_{2}$ es el coeficiente de variación $\left(L-C_{v}\right)$; $\tau_{3}$ es el coeficiente de asimetría $\left(L-C_{a}\right)$ y $\tau_{4}$ es el coeficiente de curtósis $\left(L-C_{k}\right)$.

5. Se elaboran los diagramas de Momentos-L adimensionales: $\tau_{3}$ vs. $\tau_{2}$ y $\tau_{3}$ vs. $\tau_{4}$, Figura 1 . Según Hosking y Wallis [8], una región homogénea está conformada por un grupo 
de puntos aglomerados y se representa por el centroide de dicho grupo. Los puntos que queden fuera de éste grupo acotado por una elipse son considerados discordantes. Así, un grupo conformado por varios puntos (sitios o estaciones) puede ser representado por una función de distribución.
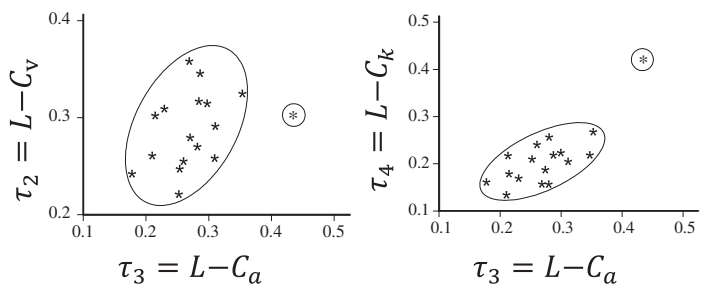

Figura 1. Diagramas de Momentos-L [8]

En aquellos casos en que existen varias conglomeraciones de puntos, estas manifiestan distintas características (Momentos- $L$ adimensionales, parámetros fisiográficos o hidrológicos, etc.), por lo tanto conforman regiones múltiples, y las elipses en los diagramas son de distintos tamaños y formas que en ocasiones no son fáciles de distinguir, porque aparecen las regiones homogéneas sobrepuestas.

Para resolver el inconveniente comentado en el párrafo anterior se utiliza el método de las $k$-Medias $[10,17]$, que permite seleccionar los sitios con características semejantes. El método logra una mejora sustancial respecto a la idea de Hosking y Wallis [8], ya que es posible incluir otras variables y la consecuente optimización en la conformación de regiones homogéneas.

\section{Método de las k-medias}

Este criterio es un algoritmo de partición que ayuda a conformar regiones de influencia o clústeres (grupos de atributos o características similares). El algoritmo busca formar grupos basado en la medición de la Distancia Euclidiana en un espacio de múltiples variables, denotada por $D_{j k}$; donde $j$ es el sitio analizado, $k$ es el sitio base o centroide $[10,17]$; cada grupo está conformado por estaciones con $p$ atributos similares a la estación base. Este método se aplica con el procedimiento secuencial siguiente:

1. Se definen $n$ grupos de variables, $x_{i j}$; donde $j$ son las estaciones; $i=1, \ldots, p$ representa el atributo considerado como relevante (Momentos- $L$, altitud, latitud, etc.).
2. Se estandarizan las magnitudes de las variables de cada atributo

$$
C_{i j}=x_{i j} / \bar{x}_{i j}
$$

Donde $\bar{x}_{i j}$ es el valor medio del atributo $i$ para todos los sitios $j$ del grupo considerado.

3. Para cada conjunto de estaciones se establecen los sitios base, $C_{i k}$, y se calculan las distancias euclidianas desde cada estación al sitio base.

$$
D_{j k}=\left[\sum_{i=1}^{p}\left(C_{i j}-C_{i k}\right)^{2}\right]^{1 / 2}
$$

En las ecuaciones anteriores, $C_{i j}$ y $C_{i k}$ son las magnitudes de las variables estandarizadas para cada atributo $i$ en las estaciones $j$ y $k$, respectivamente; donde $j=1, \ldots, m_{k} ; i=1, \ldots, p$ y $k=1, \ldots, n$.

4. Se identifican las estaciones con Distancia Euclidiana Mínima, $\min _{j} D_{j k}$, y se reasignan al grupo cuya distancia es mínima. Los nuevos sitios base se obtienen promediando los atributos de cada grupo de estaciones.

5. Se repiten los pasos 3 a 4 , hasta confirmar que no hayan reasignaciones de estaciones.

\section{Prueba de homogeneidad}

Para verificar si dos grupos de datos son homogéneos se requiere probar si provienen de la misma población; si se considera que las características estadísticas relevantes de la población son su media y su variancia, se requiere hacer las pruebas de hipótesis relacionadas con estos parámetros [16]. Si los datos de cada grupo se dividen entre su respectiva media, el promedio de los valores transformados resulta idéntica para los dos grupos e igual a la unidad, de tal forma que entonces solo se requiere probar si se puede considerar que las diferencias en las variancias de los valores transformados son menores que un cierto valor límite, en cuyo caso la hipótesis de homogeneidad no se rechaza. Para establecer el valor límite se utiliza la distribución de Fisher asociada al cociente de las variancias entre dos grupos de datos que provienen de la misma población. 


$$
F=\frac{S_{1}^{2}}{S_{2}^{2}}=\left(\frac{C_{v 1}}{C_{v 2}}\right)^{2}
$$

El valor límite para el cual se considera que no hay elementos para rechazar la hipótesis de homogeneidad es el valor de la función de distribución $F$, para una probabilidad del $90 \%, 95 \%$ o del $99 \%$ (ver Figura 2).

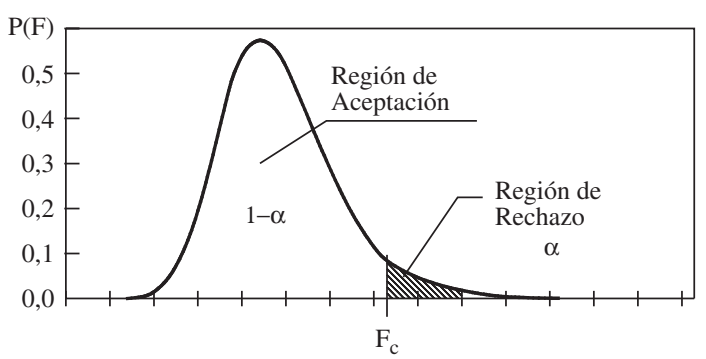

Figura 2. Función de Densidad de Probabilidad para una distribución $F$ de Fisher de cola superior.

\section{Método estaciones-año}

El método de las Estaciones-Año, también conocido como Avenida-Índice [13], considera el análisis de una muestra formada por todos los registros estandarizados de las SMA que conforman la región homogénea, $[10,16]$. En este método los datos máximos anuales como las crecidas o lluvias máximas, medidos en distintos sitios son conjugados y tratados como un solo registro. Este enfoque considera que los datos son variables aleatorias independientes, lo cual, en el caso de algunas variables no es estrictamente válido, debido a la dependencia espacial entre sus valores observados. Para lograr la homogeneidad es recomendable realizar una modificación en las variables originales, la cual consiste en estandarizar los datos, es decir, dividirlos entre su media aritmética. Los valores estandarizados generalmente presentan homogeneidad regional, mostrando un coeficiente de variación aproximadamente constante [21].

El método supone una región homogénea con $m$ sitios; donde cada sitio representa la estación de observación, $j=1, \ldots, m$, en la que se mide la $S M A, x_{i j}$. La serie estandarizada conjunta puede ser representada por una distribución de probabilidad mediante un análisis de frecuencia de la relación: $y_{i j}=x_{i j} / \bar{x}_{j}$, para todas las estaciones de la región homogénea, esto es, $y_{i j}$ se convierte en una serie de registros estandarizados, y la unión de todos los datos disponibles se considera válida para toda la región.

Un aspecto importante en este criterio es el riesgo que implica el uso de registros cortos con desigual inicio y final que las series largas, o con interrupciones en el record histórico, ya que los valores muy bajos o muy altos pueden haberse presentado durante esas interrupciones, lo que originaría sesgo en las estimaciones. La inclusión de series cortas está sujeta a una evaluación particular, pues si existen eventos importantes, estas muestras también deben ser incluidas, ya que su representación como evento regional resulta significativa $[10,12]$. De acuerdo con Escalante y Reyes [10] y Campos [12] el tamaño mínimo de la muestra de cada serie debe ser de 10 años.

A continuación se describe el procedimiento para aplicar el criterio de las estaciones-año:

1. Para cada grupo homogéneo considerado en el análisis regional se verifica la homogeneidad de los datos, como se describe en la respectiva sección.

2. Se calcula la SMA estandarizada, $y_{i j}$, mediante la ecuación que relaciona las series con sus promedios, $y_{i j}=x_{i j} / \bar{x}_{j} \quad[14,16]$.

3. Se construye la serie estaciones-año, conformada por la unión de todos los registros, $y_{i j}$.

4. Se aplica el análisis de frecuencia a la serie del paso anterior. Se elige la función de distribución de probabilidad que mejor se ajuste al registro de estaciones-año: Normal, Log-Normal, Gumbel, GVE, etc. [10, 12].

5. Una vez obtenida la función de distribución de probabilidad de mejor ajuste, se calculan valores de $y_{T}$ para distintos periodos de retorno, $T$.

6. Finalmente, los eventos de diseño para cada estación en su región y frecuencia requerida se obtienen a través de la expresión (11)

$$
\left(x_{j}\right)_{T}=\bar{x}_{j} \cdot y_{T}
$$

Donde: $\left(x_{j}\right)_{T}$ es el evento de diseño en la estación, $j$, para el periodo de retorno, $T$, en años; $\bar{x}_{j}$, es el promedio de la SMA en la estación $j ; y_{T}$, es el factor de la curva regional, obtenida del análisis de frecuencia. 


\section{APLICACIÓN}

La zona de estudio comprende varias cuencas situadas en una región del Altiplano y las Montañas de Los Andes, Bolivia, entre estas se incluyen subcuencas entorno a la Ciudad de La Paz. Geográficamente se localiza entre las coordenadas $14^{\circ} 36^{\prime}$ a $17^{\circ} 24^{\prime}$ de latitud sur y $66^{\circ} 53^{\prime}$ a $69^{\circ} 21^{\prime}$ de longitud oeste. La Figura 3 muestra la distribución de las estaciones meteorológicas.

\section{Información disponible}

La información meteorológica de la zona fue extraída de la Base de Datos Regional proporcionada por el Servicio Nacional de Meteorología e Hidrología de Bolivia (SENAMHI). La región tiene 122 estaciones, de las cuales se extrajeron todos los datos de lluvia diaria del periodo 1976-2005 utilizando las herramientas del programa HYDRACCESS [22].

De las 122 estaciones se desecharon ocho por no contar con registros de más de 10 años o debido a que los datos faltantes superaban el $10 \%$ de la extensión de la serie. En una mayoría de las estaciones se presentaban algunas fechas sin registro, por lo cual se procedió a rellenar datos y a validar las series máximas utilizando los siguientes criterios:

Para completar los registros se aplicó el método de la distancia inversa al cuadrado; condicionando a aplicar el criterio de interpolación espacial con un mínimo de tres estaciones. Las distancias de búsqueda fueron de 10 a $70 \mathrm{~km}$; ésta última sólo para aquellas estaciones con escasa información.

Una vez completadas las series se verificó que el número promedio de días con lluvia anual sea semejante al valor histórico y que la lluvia total anual y sus estadísticos tampoco difieran de los valores originales.

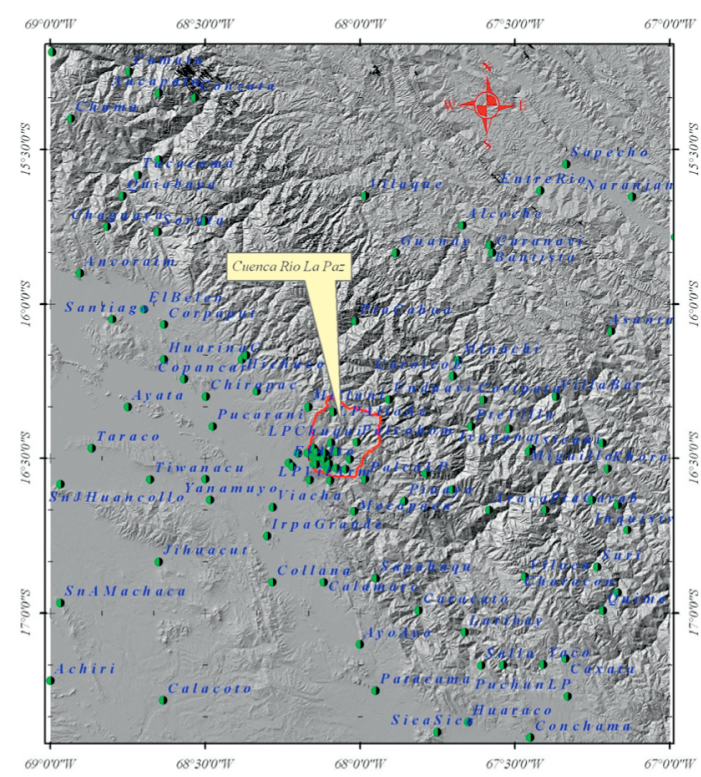

Figura 3. Ubicación de estaciones de la REM en la zona de estudio

Una vez verificadas las series de datos, se procedió a detectar y extraer las lluvias máximas diarias, clasificadas según el año hidrológico (Septiembre-Agosto).

El procedimiento para completar registros introduce eventos extremos de estaciones cercanas que probablemente no sean parte de la estación base; esto se corrige verificando las interpolaciones para la fechas en conflicto o confirmando la eliminación de la serie. De esta forma se trabajó finalmente con 114 estaciones.

En la Tabla 1 se listan las 114 estaciones, sus coordenadas, altitud, media y los Momentos-L de lluvias diarias máximas anuales. La columna mostrada como -Reg-indica la región homogénea a la que pertenece una estación, como se menciona en el acápite-Identificación de regiones homogéneas-. 
Tabla 1. Características de las estaciones meteorológicas y regiones en la zona de estudio.

\begin{tabular}{|c|c|c|c|c|c|c|c|c|c|c|c|c|c|c|c|c|c|}
\hline Estación & $\begin{array}{c}\text { Lat-S } \\
{\left[{ }^{\circ}\right]}\end{array}$ & $\begin{array}{c}\text { Long-W } \\
{\left[{ }^{\circ}\right]}\end{array}$ & $\left.\begin{array}{c}\text { ALT. } \\
{[\mathrm{msnm}]}\end{array}\right]$ & $\begin{array}{r}\bar{P}^{(1)} \\
{[\mathrm{mm}]}\end{array}$ & $\tau_{2}^{(2)}$ & $\tau_{3}^{(2)}$ & $\tau_{4}^{(2)}$ & Reg. & Estación & $\begin{array}{c}\text { Lat-S } \\
{\left[{ }^{\circ}\right]}\end{array}$ & $\begin{array}{c}\text { Long-W } \\
{\left[{ }^{\circ}\right]}\end{array}$ & $\left.\begin{array}{c}\text { ALT. } \\
{[\mathrm{msnm}]}\end{array}\right]$ & \begin{tabular}{|r|}
$\bar{P}^{(1)}$ \\
{$[\mathrm{mm}]$}
\end{tabular} & $\tau_{2}^{(2)}$ & $\tau_{3}^{(2)}$ & $\tau_{4}^{(2)}$ & Reg. \\
\hline Araca & 16,9025 & 67,5400 & 3905 & 25,4 & 0,2257 & 0,4540 & 0,3678 & 1 & LPOvejuyo & 16,5336 & 68,0472 & 3420 & 29,9 & 0,1345 & 0,2277 & 0,1986 & 2 \\
\hline & ,0942 & 68,0083 & 888 & 24,5 & 0,1449 & 0,1964 & 0,2993 & 1 & LPPan & 16,4917 & 68,1028 & 3883 & 29,8 & 0,1889 & & 854 & 2 \\
\hline & ,2806 & 68,6356 & 3830 & 23,4 & 012 & & & 1 & & , 4839 & & & 27,4 & 0,1215 & & & 2 \\
\hline apinata & 17,1336 & 66,9831 & 2976 & 36,6 & 0,1715 & 0,1053 & 0,0674 & 1 & LPPteNeg & 16,4986 & 68,1422 & 764 & 30,2 & 0,1650 & 0,1485 & 0,1142 & 2 \\
\hline Caracato & 16,9908 & 67,8111 & 2590 & 20,4 & 0,1414 & 0,1358 & 0,1578 & 1 & LPSanCali & 16,4953 & 68,1325 & 3658 & 35,0 & 0,1449 & 0,2340 & 0,1370 & 2 \\
\hline Caxata & 17,1762 & 67,3369 & 4328 & 25,5 & 0,1896 & 0,4479 & 0,4274 & 1 & non & 16,5102 & 68,1099 & 3590 & 26,7 & 0,1482 & & 0,0880 & 2 \\
\hline 1orocon & 16,8828 & 67,1497 & 2240 & 42,0 & 0,1405 & 0,2104 & 0,1680 & 1 & LPVC & 16,4828 & 68,1139 & 580 & 26,5 & 0,1235 & & & 2 \\
\hline Circuata & 16,6372 & 67,2522 & 1420 & 59,0 & 0,1847 & 0,1755 & 0,2286 & 1 & LPVTinto & 16,4808 & 68,1392 & 3865 & 33,9 & 0,1841 & ,3013 & 0,2522 & 2 \\
\hline Conchama & 17,3781 & 67,4572 & 3965 & 27,6 & 0,1254 & & 0,0966 & 1 & LPVPasan & 16,5225 & 68,14 & 3935 & 28,2 & 0,1494 & 0,0994 & 0,1562 & 2 \\
\hline Huaraco & 17,3500 & 67,6500 & 3868 & 18,9 & 0,1780 & 0,3328 & 0,4617 & 1 & Mec & 16,6711 & 68,01 & 350 & 25,6 & 0,1 & & & 2 \\
\hline Inquisiv & 16,9229 & 67,1682 & 2766 & 45,9 & 0,1706 & 0,0903 & 0,1 & 1 & Mill & 16,3333 & 68,1 & & 21,3 & & & 024 & 2 \\
\hline Isicani & 16,4761 & 67,4533 & & 64,9 & & & & 1 & & 16,5606 & & 78 & 21,9 & & & & 2 \\
\hline Khora & 16,5997 & 67,2883 & 1200 & 43,2 & 0,2035 & & & 1 & Palc & 16,4467 & 68,0 & 250 & 22,5 & 0,1322 & 865 & 371 & 2 \\
\hline Luribay & 17,0614 & 67,6619 & 2564 & 22,2 & 0,1489 & 0,0369 & 0,0376 & 1 & Pinaya & 16,6386 & 67,8 & 82 & 30,0 & 0,1 & & & 2 \\
\hline Miguilla & 16,5539 & 67,33 & 1085 & 44,8 & 0,15 & & & 1 & & 16,3 & & & 23,9 & & & 920 & 2 \\
\hline Patacama & 17,2386 & 67 & & 27,2 & & & & 1 & & 667 & & 78 & 28,3 & & & 911 & 2 \\
\hline PltaCara & 16,8156 & 67,325 & 2925 & 31,0 & 0,1701 & 0,1312 & & 1 & Taraco & 16,4667 & 68,8 & 333 & 35,0 & 0,2256 & & & 2 \\
\hline PoromaLP & 17,1711 & 67,53 & & 24,7 & & & & 1 & Tiw & 5683 & 68,6783 & 63 & 27,8 & 0,18 & & 1924 & 2 \\
\hline PuchuniL & 17,2681 & 67,3289 & 4146 & 23,1 & 0,12 & 218 & & 1 & Unc & 3100 & & 022 & 39,0 & & & & 2 \\
\hline Quime & 16,9817 & 67,219 & 3065 & 24,5 & 0,27 & & & 1 & Viac & & & 350 & 29,9 & & & 380 & 2 \\
\hline Salla & 17,1678 & 67,6081 & 35 & 26,3 & 0,1750 & & 0 , & 1 & & 15,74 & 67,6 & 640 & 57,4 & & & & 3 \\
\hline SanJacin & 16,8283 & 66,8683 & 1686 & 31,2 & 0,1532 & & & 1 & & 16,1267 & 67,1967 & 756 & 69,1 & & & & 3 \\
\hline Sapahaqu & 16,8878 & 67,95 & 31 & 22,8 & 0,20 & & & 1 & & & & 56 & 57,1 & & & & 3 \\
\hline SicaSica & 17,3333 & 67,7403 & & 26,1 & 0,2589 & 0,4177 & & 1 & Caranavi & 15,8347 & & 599 & 56,2 & & & & 3 \\
\hline Suri & 16,8500 & 67,2333 & 2516 & 52,3 & 0,19 & & 0,2017 & 1 & Chuluman & 16,4086 & 67,5 & 750 & 62,2 & 0,1881 & & 0762 & 3 \\
\hline Viloco & 16,8772 & 67,5016 & 4325 & 13,8 & 0,31 & & & 1 & Cori & 16,3 & 67,6 & 1788 & 44,7 & 0,1356 & & 0,1762 & 3 \\
\hline Yaco & 17,1658 & 67,4075 & 368 & 30,0 & 0,24 & & & 1 & Corc & 966 & & & 51,7 & & & & 3 \\
\hline Achocall & 16,5811 & 68,1608 & 3764 & 24,6 & 0,1408 & 0,2003 & 0,0677 & 2 & Covendo & 15,8300 & 66, & 50 & 84,2 & & & & 3 \\
\hline BolsaNeg & 16,5478 & 67,7875 & 4910 & 31,2 & 0,22 & & 0,3759 & 2 & EntreRio & 15,6262 & 67,4 & 30 & 83,3 & & & 956 & 3 \\
\hline Calamarc & 16,9000 & 68,1167 & 4047 & 19,4 & 0,14 & & & 2 & Gua & 15,5031 & 67,8 & 120 & 82,8 & 0,31 & & 1589 & 3 \\
\hline Chirapac & 16,2997 & 68,4981 & 38 & 22,4 & 0,2168 & 0,1751 & 0,0357 & 2 & & 16,1833 & & & 53,1 & & & 0 , & 3 \\
\hline Collana & 16,9003 & 68,2817 & & 31,9 & 0,1890 & 0,3781 & 0,3659 & 2 & Naranjan & 15,6525 & 67,1 & 375 & 68,2 & 821 & & & 3 \\
\hline Copancar & 16,2428 & 68,5686 & 3840 & 22,8 & 0,1541 & & & 2 & & 16,0561 & & 60 & 52,7 & & & & 3 \\
\hline Corpaput & 16,0688 & 68,5569 & 4033 & 21,6 & 0,0933 & 0,3858 & 0,2793 & 2 & Pue & 16,4028 & 67,6 & 225 & 42,2 & 0,2378 & & 1927 & 3 \\
\hline ElAltoAL & 16,4822 & 68,1694 & 4100 & 29,7 & 0,1939 & 0,2523 & 0,1961 & 2 & & 15,5656 & & 10 & 83,7 & 0,2445 & & 0,1878 & 3 \\
\hline ElAltoAS & 16,5103 & 68,1986 & 4034 & 32,3 & 0,1591 & 0,1756 & 0,1359 & 2 & Vilaqu & 15,6500 & 67,9 & 25 & 79,9 & 0,2600 & & 0,1571 & 3 \\
\hline ElAltoRi & 16,4833 & 68,2000 & 4080 & 30,1 & 0,20 & & & 2 & Villa & 16,3000 & 67,3 & 00 & 36,3 & & & & 3 \\
\hline ElAltoCd & 16,5308 & 68,1653 & 4040 & 27,0 & 0,1677 & 0,2308 & 0,2374 & 2 & Anco & 15,7325 & 68,5 & 4380 & 27,3 & 0,1332 & & 0,2766 & 4 \\
\hline ElAltoVi & 16,5261 & 68,2183 & 4000 & 31,6 & 0,1462 & 0,1806 & 0,1980 & 2 & Anc & 3983 & & 82 & 28,5 & & & 0,1465 & 4 \\
\hline ElBelen & 16,0164 & 68,6978 & 3833 & 24,3 & 0,1472 & 0,1220 & 0,1648 & 2 & Aucapata & 15,5000 & 68,7667 & 386 & 34,4 & 0,1583 & & 0,0578 & 4 \\
\hline Hichucot & 16,1767 & 68,3811 & 4460 & 22,9 & 0,1184 & 0,1098 & 0,0078 & 2 & Ayata & 5,2483 & & 50 & 19,2 & & & 0,2 & 4 \\
\hline HuarinaC & 16,1761 & 68,6306 & 3838 & 30,1 & 0,1847 & 0,2048 & 0,2105 & 2 & Camat & 15,2483 & 68,7450 & 2250 & 47,2 & 0,2008 & 0,1809 & 0,1878 & 4 \\
\hline Irupana & 16,4731 & 67,4528 & & 55,4 & 0,1320 & 0,1702 & 0,2648 & 2 & Carab & 15,7589 & 69,0 & 28 & 22,8 & 0,1561 & 0,1186 & 0,1518 & 4 \\
\hline Jihuacut & 16,8581 & 68,6622 & & 19,8 & 0,1776 & 0,1154 & 0,3283 & 2 & Chaguaya & 15,7500 & 68,8167 & 305 & 25,1 & 0,1975 & & 0,0709 & 4 \\
\hline Lambate & 16,6052 & 67,7032 & 365 & 33,6 & 0,1378 & 0,1 & & 2 & Chara & 15,1954 & 69,0 & 3630 & 13,3 & & & 0,0795 & 4 \\
\hline LPAchach & 16,4669 & 68,1533 & 3840 & 29,6 & 0,1677 & 0,2623 & 0,1659 & 2 & Chuma & 15,4000 & 68,9333 & 3000 & 32,2 & 0,2389 & 0,3816 & 0,2114 & 4 \\
\hline LPAchuma & 16,5308 & 68,0714 & 3384 & 24,5 & 0,2031 & 0,3728 & 0,2986 & 2 & Conzata & 15,3333 & 68,5 & 05 & 28,2 & 0,1224 & 0,2 & 0,1892 & 4 \\
\hline LPAltoAc & 16,3506 & 68,0867 & 4380 & 23,1 & & 0,0794 & 0,0452 & 2 & Curupam & 5,8333 & 68,6 & 3560 & 30,2 & 0,2069 & & 0,0526 & 4 \\
\hline LPAltoOb & 16,5236 & 68,1111 & 3370 & 25,2 & 0,1801 & 0,2743 & 0,2 & 2 & Hilo & & 69, & 4000 & 25,8 & & & 0,2334 & 4 \\
\hline LPAltoSe & 16,5347 & 68,1056 & 3384 & 24,4 & 0,1159 & 0,0176 & 0,0114 & 2 & Italaque & 15,4775 & 69,0415 & 3500 & 30,5 & 0,1827 & 0,3065 & 0,2777 & 4 \\
\hline LPBarrio & 16,5236 & 68,1014 & 3410 & 25,4 & 0,1386 & 0,1780 & 0,1886 & 2 & Puina & 14,6022 & 69,1208 & 732 & 24,9 & 0,1805 & 0,2049 & 0,1781 & 4 \\
\hline $1 \mathrm{laV}$ & 16,53 & & & 24,7 & & & & 2 & & & & & 35,9 & & & & 4 \\
\hline hican & 16,4822 & 68,0767 & 3620 & 24,1 & & 0,2 & & 2 & & & & 3845 & 23,3 & & & 0,2028 & 4 \\
\hline LPChuqui & 16,4497 & 68,0925 & 4024 & 32,0 & 0,1659 & 0,2374 & 0,0730 & 2 & Sorata & 15,7667 & 68,6517 & 2695 & 35,8 & 0,2761 & 0,4682 & 0,4125 & 4 \\
\hline LPEITeja & 16,4928 & 68,1539 & 3780 & 32,3 & 0,2009 & 0,3067 & 0,2232 & 2 & Suches & 14,8167 & & 4594 & 22,8 & 0,1680 & 0,3917 & 0,4595 & 4 \\
\hline & & & & 28,3 & & & & 2 & & & & & 29,3 & & & 1766 & 4 \\
\hline LPMallas & 16,5708 & 68,0964 & 3280 & 23,3 & 0,1273 & 0,2969 & 0,2362 & 2 & VillaPun & 15,6664 & . 69,1672 & 3835 & 27,5 & 0,1802 & 0,3274 & 0,3140 & 4 \\
\hline
\end{tabular}




\section{Características de las estaciones meteorológicas}

Las características principales de las SMA corresponden a los estadísticos de la precipitación máxima diaria obtenidos mediante los Momentos- $L$, y las características geográficas (como la altitud y coordenadas geográficas de cada sitio), ver Tabla 1. Estos parámetros varían en un amplio rango de valores, y reflejan características meteorológicas propias de las estaciones y de la zona que las limita. Algunos eventos máximos se deben a sucesos ocurridos en toda la región, por tanto es razonable incluir valores representativos de cada sitio para lograr una regionalización basada en la clasificación de los Momentos- $L$ de la precipitación, así como la altitud y su posición geográfica.

Es interesante ver que la topografía de la región es muy accidentada, con altitudes que van de 375 a 4510 msnm (ver Figura 3 y Tabla 1). Las estaciones de la zona interandina se encuentran entre 2000 y $3800 \mathrm{msnm}$; las del altiplano de 3800 a 4000 msnm, y en las montañas de Los Andes por encima de los $4000 \mathrm{msnm}$; en estas zonas hay sitios entre las montañas con altitudes mucho menores y probablemente por ello la correlación exclusiva "altitud-precipitaciones máximas" es poco significativa para el análisis regional, ya que definen áreas dispersas, como se muestra en la Figura 4. Finalmente, las estaciones de valles y las llanuras amazónicas están abajo de los 2000 msnm.

\section{Identificación de regiones homogéneas}

Se examinaron distintos atributos, entre estos: la magnitud de la precipitación diaria máxima anual, los momentos- $L$, la altitud sobre el nivel del mar y coordenadas UTM de las estaciones. Con éstas características se aplicó el criterio de regionalización descrito anteriormente, y se encontró que las variables más significativas son el coeficiente de variación, $\tau_{2}=L-C_{v}$ y la altitud. Al incluir las coordenadas de las estaciones en el análisis se conforman regiones geográficas homogéneas.

\section{Análisis de frecuencia regional de lluvias}

En la bibliografía se cuenta con varios métodos de análisis de frecuencia, así mismo existen diversos criterios de solución de éstos [10]. En el actual trabajo se han empleado preferentemente las funciones de distribución de probabilidad (FDP) para eventos extremos, como la GVE y la Gumbel Mixta. La mayoría de las SMA se ajustan muy bien a la FDP
Gumbel, lo cual muestra que es buena aproximación local y regional, pero su dispersión y los eventos más grandes (outliers) cambian la tendencia natural de algunos datos; generando el comportamiento típico de una SMA de dos poblaciones.

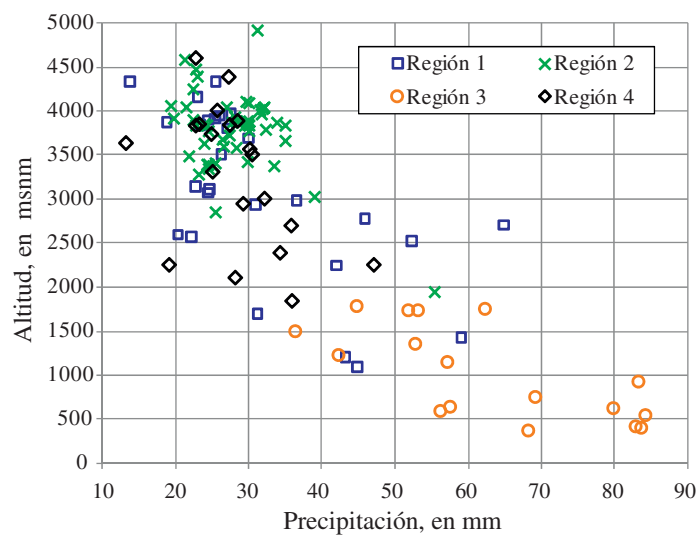

Figura 4. Relación de lluvia diaria máxima promedio anual contra altitud.

Mendoza [4] y Luna [5] encontraron que los registros de lluvia pluviográfica en la región se ajustan a FDP de tipo GV1 (Gumbel), y en general, las SMA diarias analizadas aquí también tienen ese comportamiento. Por consiguiente, los resultados del análisis de frecuencia están basados en ajustes probabilísticos de esas funciones.

\section{Conformación de regiones mediante diagramas híbridos de momentos-L}

Como se dijo, varios autores sugieren conformar regiones homogéneas con los Momentos-L, pues al ser aplicados como se indica en la sección correspondiente las regiones homogéneas están definidas por los diagramas de Momentos $-L$ adimensionales sugeridos por Hosking y Wallis [8]. Sin embargo, la zona en cuestión presenta diagramas como el de la Figura 5, en los que es complejo definir una o más regiones homogéneas. Así, en el diagrama inferior ( $\tau_{3}$ vs. $\tau_{4}$ ), se han incluido las FDP teóricas; el trazo continuo corresponde a distintas combinaciones de parámetros de una FDP GVE y el punto corresponde al caso particular de la Gumbel. Todos los conjuntos de puntos próximos a la distribución teórica representan regiones homogéneas que logran la estimación regional de los eventos. La figura de arriba ( $\tau_{3}$ vs. $\tau_{2}$ ) es una correspondencia de la otra, puesto que conserva homogeneidad en sus estadísticos. 

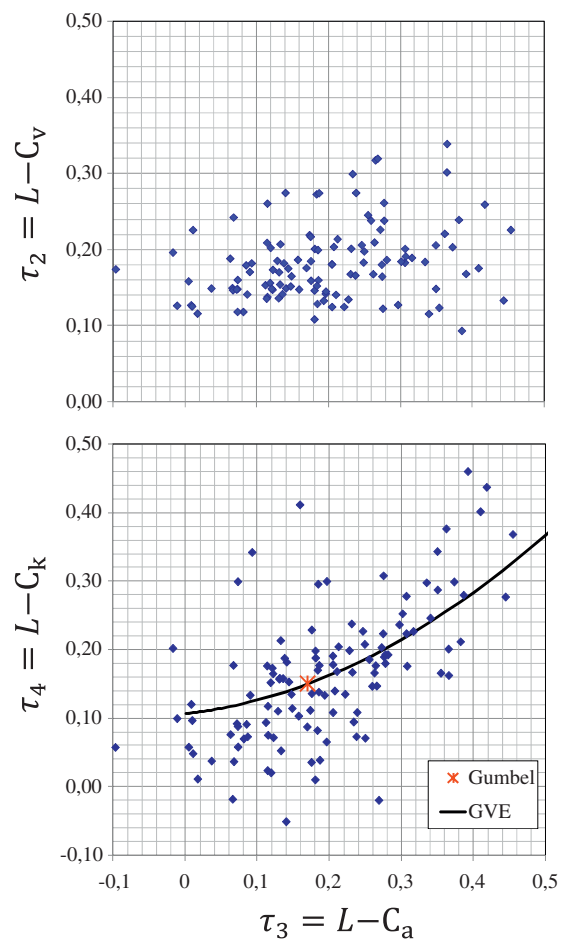

Figura 5. Diagramas de Momentos-L con datos de la región de estudio.

Si se desea aplicar el concepto formulado por Hosking y Wallis [8], descrito en el párrafo anterior, la conformación de regiones homogéneas no es muy fácil. Una combinación favorable surge al aplicar el método de las $k$-medias, definiendo como variables importantes a los Momentos $-L$ y a las características geográficas de las estaciones.

La Figura 6 presenta el diagrama híbrido con Momentos $-L$ (en particular $\tau_{3}=L-C_{v}$ ), la altitud y las coordenadas geográficas, las cuales fueron necesarias para definir 4 regiones con características homogéneas. En la parte superior se presenta una vista tridimensional, con el coeficiente de variación en el eje vertical y las coordenadas de las estaciones en el plano horizontal. Se puede apreciar que los puntos de cada región y su respectivo centroide forman nubes relativamente cercanas entre sí. En la parte inferior de la Figura 6 se aprecia una vista de la conglomeración de las cuatro regiones.
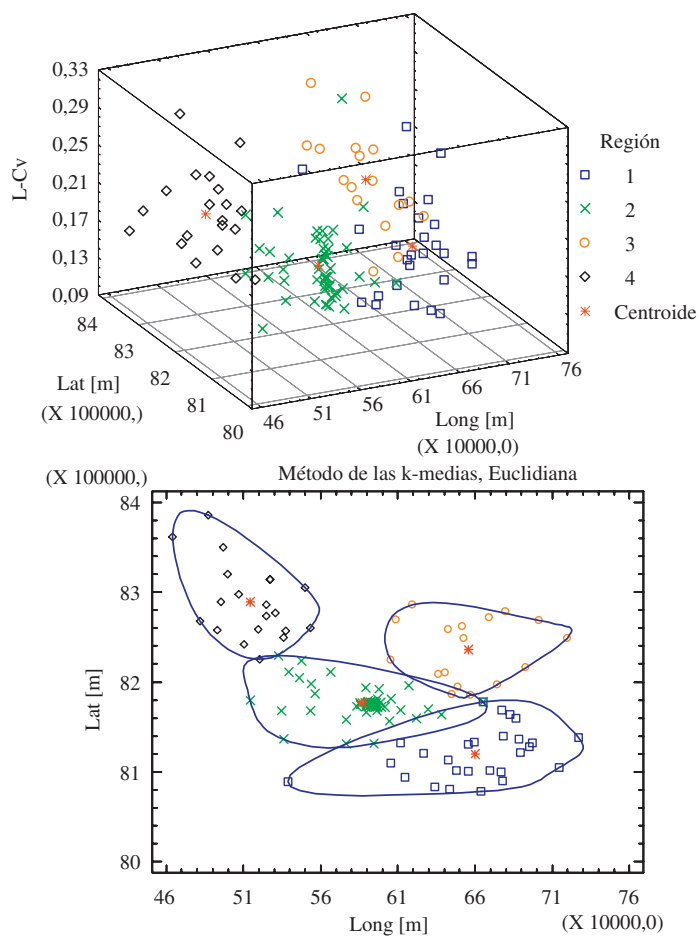

Figura 6. Diagramas híbridos de momentos-L que muestran regiones homogéneas, obtenidas al agrupar características similares de acuerdo con el método de las $k$-Medias con distancias Euclidianas.

En la Tabla 2 se indica la cantidad de miembros (estaciones) que componen cada región y un resumen de los parámetros que caracteriza al centroide correspondiente.

Tabla 2. Parámetros que representan centroides de las cuatro regiones homogéneas

\begin{tabular}{|c|c|c|c|c|c|c|}
\hline $\begin{array}{c}\text { Clúster } \\
\text { o Región }\end{array}$ & $\begin{array}{c}\text { No. } \\
\text { Est. }\end{array}$ & $\%$ & $\begin{array}{c}\tau_{2} \\
{[\text { adim }]}\end{array}$ & $\begin{array}{c}\text { ALT } \\
{[\mathrm{msnm}]}\end{array}$ & $\begin{array}{c}\text { LAT } \\
{\left[{ }^{\circ}\right]}\end{array}$ & $\begin{array}{c}\text { LONG } \\
{\left[{ }^{\circ}\right]}\end{array}$ \\
\hline 1 & 27 & 23,68 & 0,1867 & 3079,41 & 17,0013 & 67,4953 \\
\hline 2 & 50 & 43,86 & 0,1632 & 3765,78 & 16,4895 & 68,1898 \\
\hline 3 & 17 & 14,91 & 0,2247 & 1032,94 & 15,9486 & 67,5457 \\
\hline 4 & 20 & 17,54 & 0,1995 & 3278,00 & 15,4703 & 68,8661 \\
\hline
\end{tabular}

\section{ESTIMACIÓN REGIONAL}

Una vez definidos los grupos homogéneos (Figura 8), se utilizan las SMA de las estaciones que caen en cada región, se estandarizan respecto a la media de cada estación y se forman las series estaciones-año para cada una de las regiones; a continuación, se aplica el análisis regional de frecuencias a cada 
serie y se obtiene el mejor modelo de ajuste. En la Figura 7 se presenta una comparación gráfica entre las FDP teóricas y la FDP empírica, dada por la fórmula de Weibull, $T=(n+1) / m$, donde $T$ es periodo de retorno, $n$ número de datos, y $m$ orden de la serie.
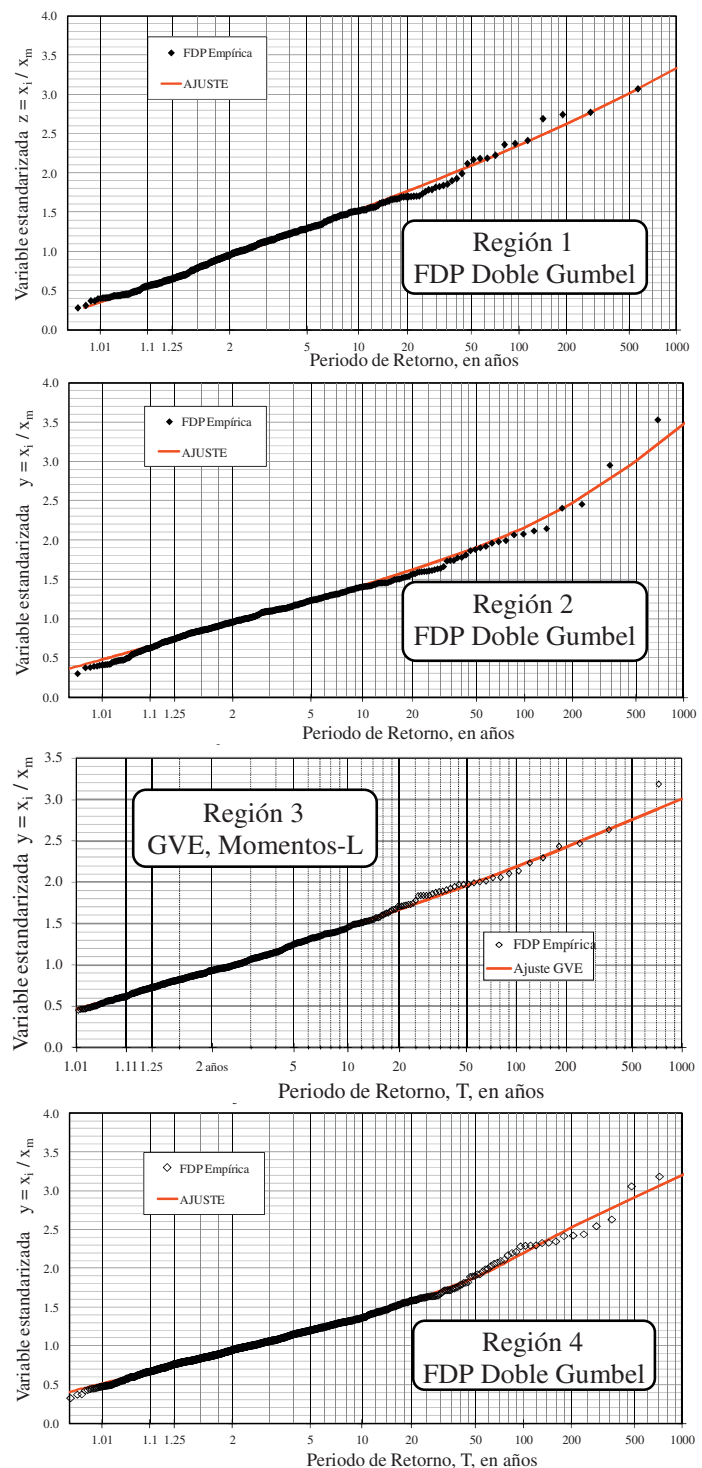

Figura 7. Resultados del análisis de frecuencia regional.

Los eventos de diseño, $\left(x_{j}\right)_{T}$, para el periodo de retorno deseado, $T$, se obtienen al multiplicar el promedio de la SMA, $\bar{x}_{j}$, considerada en algún sitio de una región predeterminada, por el factor regional, $y_{T}$, aportados en la Tabla 3 .
Tabla 3. Factores regionales $y_{T}$ para las regiones homogéneas 1, 2, 3, y 4.

\begin{tabular}{|c|c|c|c|c|c|c|}
\hline \multirow{2}{*}{$\begin{array}{c}\text { T } \\
\text { [años] }\end{array}$} & \multirow{2}{*}{$\boldsymbol{p}^{(\mathbf{1})}$} & \multirow{2}{*}{$\boldsymbol{Z}^{(\mathbf{2 )}}$} & \multicolumn{4}{|c|}{$y_{T}=\left(x_{j}\right)_{T} / \bar{x}_{j}$} \\
\cline { 4 - 7 } & & & Reg.1 & Reg.2 & Reg.3 & Reg.4 \\
\hline 1000 & 0,999 & 6,9073 & 3,330 & 3,472 & 3,009 & 3,197 \\
\hline 500 & 0,998 & 6,2136 & 3,009 & 3,001 & 2,749 & 2,917 \\
\hline 200 & 0,995 & 5,2958 & 2,620 & 2,467 & 2,422 & 2,523 \\
\hline 100 & 0,99 & 4,6001 & 2,348 & 2,156 & 2,185 & 2,194 \\
\hline 50 & 0,98 & 3,9019 & 2,091 & 1,903 & 1,957 & 1,878 \\
\hline 20 & 0,95 & 2,9702 & 1,767 & 1,618 & 1,666 & 1,573 \\
\hline 10 & 0,9 & 2,2504 & 1,528 & 1,420 & 1,452 & 1,384 \\
\hline 5 & 0,8 & 1,4999 & 1,285 & 1,225 & 1,238 & 1,203 \\
\hline 2 & 0,5 & 0,3665 & 0,928 & 0,941 & 0,932 & 0,941 \\
\hline 1,25 & 0,2 & $-0,4759$ & 0,668 & 0,734 & 0,717 & 0,750 \\
\hline 1,1111 & 0,1 & $-0,8340$ & 0,558 & 0,646 & 0,629 & 0,669 \\
\hline 1,0101 & 0,01 & $-1,5272$ & 0,347 & 0,478 & 0,463 & 0,512 \\
\hline 1$)$ & $p=(T-1) / T,{ }^{2)} Z=-\ln (-\ln p)$ & \\
\hline
\end{tabular}

\section{ANÁLISIS DE RESULTADOS}

Con la finalidad de verificar la bondad de la metodología propuesta se realizó el cálculo de eventos para cada estación ubicada en las regiones geográficas de la Figura 8, primero con un modelo de FDP individual y luego aplicando el modelo regional propuesto. La columna Reg. en la Tabla 1 indica la pertenencia de cada estación con la región $1,2,3$ ó 4.

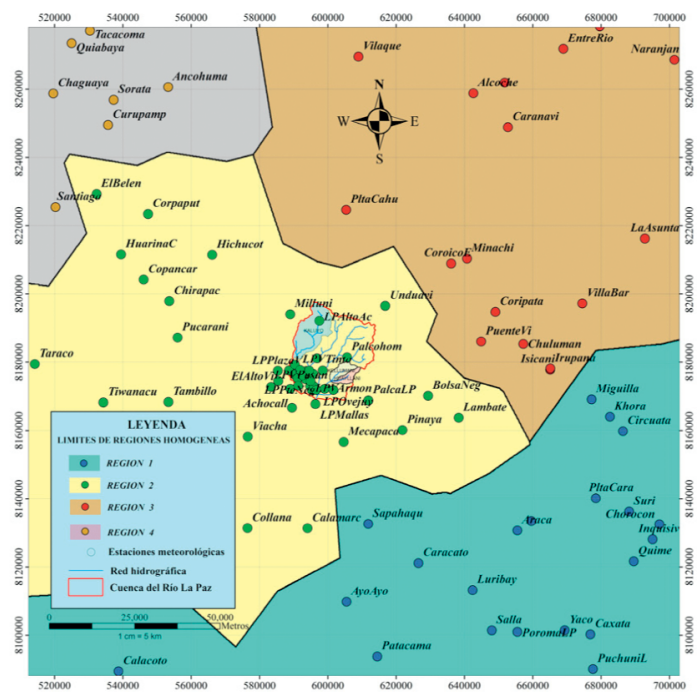

Figura 8. Regiones homogéneas de SMA de lluvia diaria para la zona de estudio. 

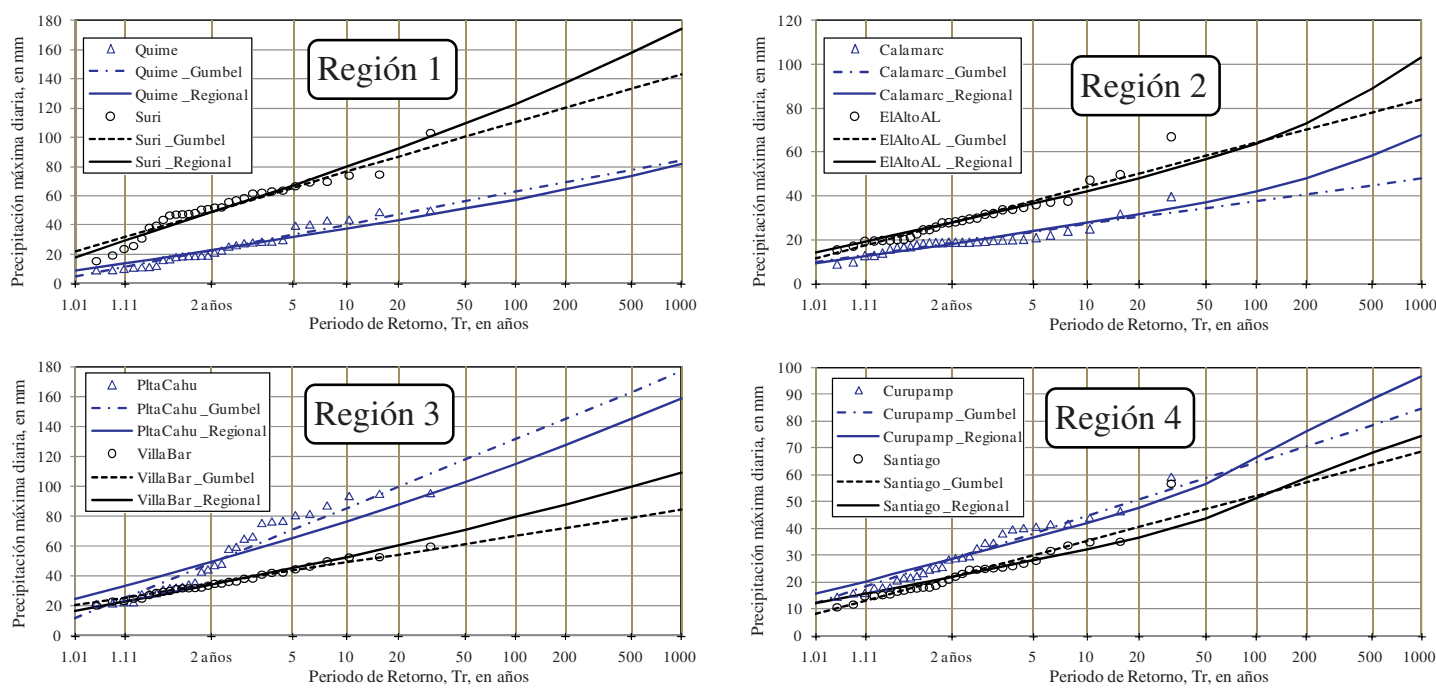

Figura 9. Comparación de FDP ajustadas individualmente y las obtenidas por Ajuste Regional para algunas SMA de la región de estudio.

La Figura 9 presenta comparaciones de las predicciones de precipitación para unas cuantas estaciones de cada región. Cada gráfico contiene las SMA de dos estaciones por región, el ajuste con la FDP obtenida individualmente (Gumbel) y el contraste con el ajuste regional aplicando los factores de la Tabla 3.

En la Tabla 4 se comparan los valores calculados con el análisis puntual y el regional, obtenidos para algunas estaciones de las cuatro regiones. Los valores de dicha tabla presentan un cambio en la tendencia de eventos mayores a 50 años, situación que se evidencia en las series con mayor información; por ejemplo, las estaciones El Alto y San Calixto (Figura 10), registraron eventos extremos importantes y transfieren esta información a las estaciones cercanas. Este efecto es importante al momento de realizar un análisis particular en cada caso; así el ajuste individual con la FDP Gumbel predice resultados menores a los registrados en la región homogénea. La Figura 10 muestra un ejemplo de este caso; primero, en la estación El Alto el modelo regional estima los eventos grandes mejor que un análisis puntual. Luego, en la estación San Calixto, con 88 años de registro, el modelo individual GVE-I se ajusta mejor a la serie extensa, pero al hacer la estimación regionales evidente que la estación recibe información de las estaciones vecinas.
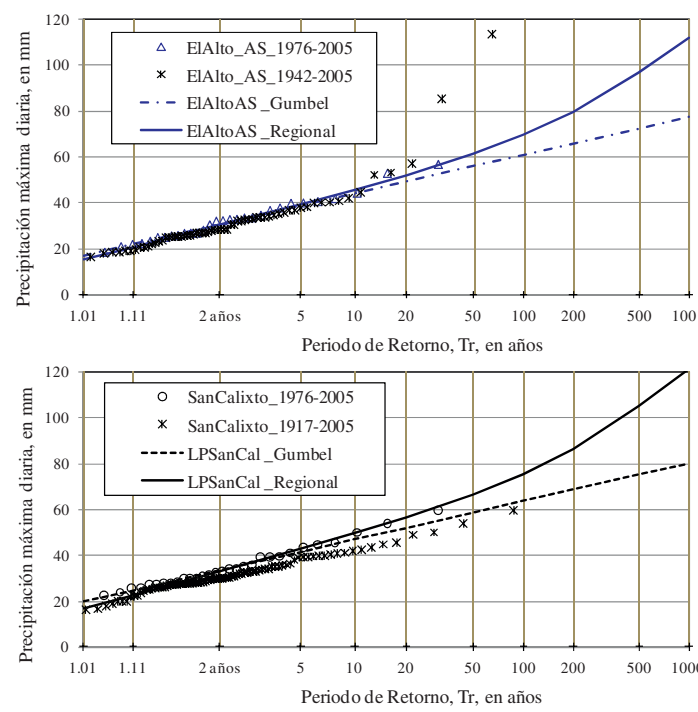

Figura 10. Comparación con estaciones de mayor record de información.

\section{Prueba de homogeneidad}

Se aplicó la prueba de Fisher a las series de las regiones conformadas, y con base en el parámetro crítico, $F_{c}(\alpha, N, M)$, se verificó que la mayoría de las estaciones en cada zona están en la región de aceptación: $F=\left(L-C v_{1} / L-C v_{2}\right)^{2} \leq F c$, con $\alpha=5 \%$; $N$ y $M$ son las longitudes de las SMA, en este caso se supone el caso extremo cuando se tiene poca información. 
Tabla 4. Comparación de lluvia diaria máxima anual estimadas con análisis puntual y regional.

\begin{tabular}{|c|c|c|c|c|c|c|c|c|c|c|}
\hline & \multicolumn{3}{|c|}{$\mathrm{T}$ [años] $=$} & 2 & 5 & 10 & 20 & 50 & 100 & 500 \\
\hline & \multicolumn{3}{|c|}{ Probabilidad $=$} & 0,5 & 0,8 & 9 & 95 & 98 & 0 & \\
\hline & & & FDP & \multicolumn{7}{|c|}{ SMA de lluvia diaria, $P$, en $\mathrm{mm}$} \\
\hline \multirow[t]{2}{*}{1} & & Araca & REG. & 23,6 & 32,7 & 38,8 & 44,9 & 53,2 & 59,7 & 76,5 \\
\hline & & Araca & $\mathrm{m}$. & $23,4 \mid$ &, 5 & & & & & \\
\hline \multirow[t]{2}{*}{5} & & & & 18,9 & 26,2 & & & & & \\
\hline & & & Gum. & 19,5 & & & & & & 42,9 \\
\hline \multirow[t]{2}{*}{10} & & Hua & REG. & 17,5 & 24,2 & 8 & & 39,4 & 44,2 & 56,7 \\
\hline & & Hua & Gum. & 17,5 & 24,7 & & & & & 54,4 \\
\hline \multirow[t]{2}{*}{15} & & Mig & REG. & 41,6 & 57,6 & & & & 105 & 135 , \\
\hline & 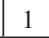 & Mig & & 42,7 & 54,2 & & & & & 102 , \\
\hline \multirow[t]{2}{*}{20} & 1 & & REG. & 22,7 & 31,5 & & & & & 73,7 \\
\hline & & Qui & Gum. & 22,5 & 33,2 & &, 1 & & 5 & 77,7 \\
\hline \multirow[t]{2}{*}{25} & & & REG. & 48,6 & 67,2 & & 4 & & & 157, \\
\hline & & $\mathrm{Su}$ & Sum. & 49,3 &, 6 & & & & & 133 , \\
\hline \multirow[t]{2}{*}{30} & 2 & $\mathrm{Cal}$ & REG. & 18,3 & 23,8 & & & & & 58,3 \\
\hline & 2 & $\mathrm{Cal}$ & Gum. & 18,5 & 23,6 & & & & & 45,0 \\
\hline \multirow[t]{2}{*}{35} & 2 & & REG. & 27,9 & 36,4 & & & & & 89,2 \\
\hline & 2 & ElA & Gum. & 27,9 & 37,6 & & & & & 77,9 \\
\hline \multirow[t]{2}{*}{40} & 2 & & & 22,9 & 29 & & & & & 72,9 \\
\hline & 2 & ElB & Gum. & 23,2 & 28,9 & & & & & 52,6 \\
\hline \multirow[t]{2}{*}{45} & 2 & & REG. & 31,6 & 41,1 & & & & 4 & 100, \\
\hline & & & Gum. & 32,2 & 39,4 & & & & & 68,9 \\
\hline \multirow[t]{2}{*}{50} & 2 & LPA & REG. & 22,9 & 29,9 & & & & & 73,2 \\
\hline & 2 & & Gum. & 23,6 & 27,9 & & & & & 45,8 \\
\hline \multirow[t]{2}{*}{55} & & & & 30 & & & & & & 97,0 \\
\hline & 2 & LPI & Gum. & 30,2 & 41,5 & & 56 & & 72 & 88,2 \\
\hline \multirow[t]{2}{*}{60} & 2 & & EG. & 25,8 & 33,5 & & & & & 2,2 \\
\hline & 2 & & Gum. & 26,3 & 0 & & & & & 55,6 \\
\hline 65 & 2 & & REG. & 31,9 & 41,6 & & 54,9 & 64,6 & 73,2 & 101, \\
\hline & 2 & & Gum. & 32,0 & & & & & & 87,1 \\
\hline 70 & 2 & & REG. & 21,2 & 27,6 & & & & & 67, \\
\hline & 2 & Palc & Gum. & 21,6 & 26,3 & & & & & 45,8 \\
\hline & & & & 26,1 & & & & & & 83,4 \\
\hline & 2 & Tiw & Gum. & 26,1 & 35,0 & & 46,6 & 53,9 & 59 & 72,0 \\
\hline 80 & 3 & & REG. & 53,2 & 70,6 & & & & & 156, \\
\hline & 3 & $\mathrm{Bau}$ & Gum. & 53,7 & 71,9 & & & & 7 & 147, \\
\hline 85 & 3 & & EG. & 78,4 & 104,2 & & & 16 & 184 & 231, \\
\hline & 3 & Cov & Gum. & 80,1 & & & & & & 194, \\
\hline 90 & 3 & & REG. & 49,1 & 65,2 & & & & & 144, \\
\hline & 3 & Plta & Gum. & 48,5 & 70,7 & & & & 5 & 163, \\
\hline 94 & 3 & & G. & 33,8 & & & & & & 99,8 \\
\hline & 3 & Villa & Gum. & 34,7 & 43,3 & & 54,4 & & 66,7 & 78,9 \\
\hline 95 & 4 & Anc & REG. & 25,7 & 32,9 & & & & & 79,7 \\
\hline & 4 & & Gum. & 26,1 & 32,8 & & 41, & & & 60,4 \\
\hline & 4 & & REG. & 21,5 & 27,5 & & 35,9 & & \begin{tabular}{|l|}
50,1 \\
\end{tabular} & 668 \\
\hline & 4 & Cara & Gum. & 21,8 & 27,5 & & 34, & 39 , & 43,1 & 51,2 \\
\hline & 4 & & REG. & 28,4 & 36,3 & & 47,5 & & 66,3 & 88,1 \\
\hline & 4 & Cur & Gum. & 28,4 & 38,1 & & & & & 78, \\
\hline 10 & 4 & & REG. & 21,9 & 28,0 & & 36,6 & & & $68,($ \\
\hline & 4 & Sant & Gum. & 21,8 & 29,9 & & 40,4 & & 52,1 & 63,6 \\
\hline & 4 & & & & & & & & & 80,1 \\
\hline & 4 & Villal & Gum. & 25,8 & 34,6 & 4 & 46,0 & 53,2 & 58,6 & 71, \\
\hline
\end{tabular}

\section{CONCLUSIONES}

La región de estudio tiene una red con poca densidad de estaciones, pero el análisis regional coadyuva a que las estaciones de gran amplitud de datos transfieran información a sitios que no la tienen o sólo tienen pocos registros, de tal manera que el análisis regional logra una mejora en la estimación de eventos extremos para la zona de estudio.

Al emplear las características de las SMA, como los Momentos- $L$; con la altitud y coordenadas geográficas, se ha logrado delimitar cuatro regiones homogéneas y se han obtenido factores regionales de diseño, que permiten la estimación de eventos extremos de lluvia diaria máxima anual en cualquier sitio.

A partir de la Figura 8 se observa que la región 1 comprende el altiplano y subcuencas del sur; en cambio, la región 2 está definida por gran parte de la cuenca del Río La Paz y el altiplano medio. La región 3 abarca zonas geográficas de los llanos y pie de montaña. Por último, la región 4 comprende las montañas, el altiplano norte y sectores del Lago Titicaca.

Las Figuras 9 y 10 muestran que, al conformar regiones homogéneas, las estimaciones de lluvias de diseño son más confiables que las obtenidas con el análisis individual por estaciones, sobre todo para periodos de retorno grandes. Esta ventaja es más clara en el caso de estaciones sin datos o con poca información. Adicionalmente, los resultados del análisis regional se pueden utilizar para realizar estimaciones de lluvias para el diseño en sitios no instrumentados, para lo cual únicamente se requiere identificar el sitio en alguna de las regiones y estimar la media de precipitaciones diarias máximas anuales de las estaciones vecinas; luego, por interpolación se calcula el valor puntual en el sitio de interés. Finalmente, se aplican los factores regionales y se estima la lluvia para un periodo de retorno.

Una aplicación práctica con los resultados presentados es calcular el caudal pico de una crecida o su hidrograma. Esto se logra, primero, estimando la duración de la tormenta, $d$, para la cuenca de interés; que para cuencas pequeñas es aproximadamente igual al tiempo de concentración, $d=t_{c}$ [2]. Luego, se obtiene el cociente empírico de lluvias, $K_{d}=P_{T}^{d} / P_{24}^{T}$, con base en resultados de estudios 
locales [4-5] y para el periodo de retorno deseado. De esta forma, se puede calcular la lluvia total en términos de la lluvia diaria, $P_{24}^{T}$, y $K_{d}$, es decir: $P_{T}^{d}=K_{d} \cdot P_{24}^{T}$.

Luego, para obtener un hietograma sintético se aplica el criterio de distribución temporal propuesto por Luna y Domínguez [23], quienes plantean disgregar las tormentas de corta o larga duración. Una vez que se tiene la lluvia total o el hietograma se puede aplicar un modelo de lluvia-escorrentía para estimar el hidrograma en la cuenca de estudio.

Por último, conviene recalcar que al contar con estimaciones más confiables de las precipitaciones de diseño se logrará también una mejor estimación de crecidas en la zona.

Los resultados logrados en este estudio han sido desarrollados con datos de la red meteorológica de la región, proporcionados por el Servicio Nacional de Meteorología e Hidrología de Bolivia. El presente aporte otorga un conocimiento para la predicción de eventos extremos en la región, a fin de que los proyectistas incluyan el método y resultados en el diseño hidrológico. Del mismo modo, se espera que la metodología sea replicada en toda la red meteorológica del país.

En estudios posteriores se recomienda actualizar la información para tomar en cuenta eventos extremos de recientes años.

Se ha demostrado que la información meteorológica es muy valiosa para cuantificar eventos extremos y los relacionados con el manejo de los recursos hídricos. A pesar de que la región estudiada tiene una REM poco densa, los resultados hallados proporcionan información confiable para el diseño de obras hidráulicas; en cambio, en otras regiones del país la densidad de las redes hidrometeorológicas es mucho menor, por tanto es recomendable instalar estaciones en sitios definidos estratégicamente para lograr resultados favorables en la modelación meteorológica e hidrológica.

\section{AGRADECIMIENTOS}

El presente trabajo fue elaborado con apoyo del Instituto de Ingeniería de la Universidad Nacional Autónoma de México.
Se agradece la información proporcionada al Servicio Nacional de Meteorología e Hidrología de Bolivia y a sus funcionarios ingenieros: Carlos Díaz, Luis Noriega, Javier Mendoza y Virginia Chávez.

\section{REFERENCIAS}

[1] USDA. "Urban Hydrology for Small Watersheds". Engineering Division, Soil Conservation Service, US Department of Agriculture, Washington, DC Tech. Rep. 55. USA. URL: ftp://ftp.wcc.nrcs.usda.gov.1986.

[2] V.T. Chow, D. Maidment y L. Mays. "Hidrología Aplicada". Mc Graw Hill, Traducido de la primera edición en inglés de Applied Hydrology, p. 585. Bogotá, Colombia. 1994. ISBN: 958-600-171-7.

[3] A.D. Feldman; US Army Corps. "Hydrologic Modelling System HEC-HMS, Technical Reference Manual". Hydrologic Engineering Center. Davis, p. 157. California, USA. 2000.

[4] J.C. Mendoza R. "Estudio del Régimen Pluviométrico en la Cuenca del Río La Paz". Proy. de Grado Ingeniería Civil. Facultad de Ingeniería. Universidad Mayor de San Andrés. Bolivia. 1994.

[5] J.A. Luna V. "Análisis de Ecuaciones para la Relación Precipitación-DuraciónFrecuencia”. Recursos Hídricos. IHH-UMSA. Bolivia. Vol. $1 \mathrm{~N}^{\circ}$ 2, pp. 58-71. Agosto 2002. [6] J.R. Hosking, J.R. Wallis and E.F. Wood. "Estimation of the Generalized Extreme- Value Distribution by the Method of Probability Weigthed Moments". Technometrics. Vol. 27, Issue 3, pp. 251-261. August, 1985. ISSN: 00401706.

[7] J.R. Hosking.'L - moments: analysis and estimation of distribution using linear combinations of order statistics". Journal of the Royal Statistical Society. Vol. 52, Issue 1,pp. 105-124. 1990. ISSN: 00359246.

[8] J.R.M. Hosking y J.R.Wallis. "Regional Frequency Analysis - an Approach Based on L-Moments". Cambridge University Press, 1ra Ed., p. 224. Cambridge, UK. 1997. ISBN: 0-521-43045-3.

[9] J.R. Stedinger, R.M. Vogel and E.FoufoulaGeorgiou. "Frequency Analysis of Extreme Events". En Handbook of Hydrology. C.18. Ed. D. Maidment-Mc Graw Hill, pp. 18.118.66. NY, USA. 1993. ISBN: 0070397325. 
[10] C.A. Escalante S. y L. Reyes Ch. "Técnicas Estadísticas en Hidrología”. Facultad de Ingeniería. Universidad Nacional Autónoma de Madrid. 2da Ed., p. 298. 2005.

[11] R.M. Vogel and N.M. Fennessey. "L-Moment Diagrams Should Replace Product Moment Diagrams". Water Resources Research. Vol. 29, Issue 6, pp. 1745-1752. June, 1993. ISSN 0043-1397.

[12] D.F. Campos A. "Análisis Probabilístico Univariado de Datos Hidrológicos". AMHIMTA, Avances en Hidráulica 13, pp. 133-161. México, DF. 2006. ISSN 1405-7743.

[13] T. Dalrymple. "Flood Frequency Methods". U.S. Geological Service. Water Supply. Vol. 1543(A), pp. 11-51. 1960. URL: http://pubs. er.usgs.gov/

[14] R. Domínguez M., C. Bouvier, L. Neppel, and H. Lubes. "Approche régionale pour l'estimation des distributions ponctuelles des pluies journalières dans le LanguedocRoussillon (France)". Hydrological Sciences Journal. Vol. 50, Issue 1, pp. 17-29. 2005. ISSN 0262-6667.

[15] D.F. Campos A. "Ajuste Regional de la Distribución GVE en 34 Estaciones Pluviométricas de la Zona Huasteca de San Luis Potosî́”. Agrociencia, pp. 57-70. México. 2008. ISSN 1405-3195.

[16] Sandra E. Palacios C. "Tormentas de Diseño en el Río Grijalva-México". Tesis de Maestría. Facultad de Ingeniería. Universidad Nacional Autónoma de México.México. 2010.

[17] Y. Lihua and D. Meilan. "Based on k-means and fuzzy k-means algorithm classification of Precipitation". International Symposium on Computational Intelligence and Design. IEEE Computer Society, pp. 218-221. 2010. ISSN 978-0-7695-4198-3.

[18] I.J. Pelczer and H.L. Cisnéros-Iturbe. "Identification of rainfall patterns over the Valley of Mexico". 11th International Conference on Urban Drainage. Edinburgh, Scotland, UK. pp. 1-9, 2008.

[19] C. Cunnane. "Methods and merits of regional flood frequency analysis". Journal of Hydrology. B.V. Amsterdam: Elsevier Science Publishers. Vol. 100, pp. 269-290. 1988. ISSN: 0022-1694.

[20] J.A. Greenwood, J. Maciunas Landwehr N, C. Matalas and J. R. Wallis. "Probability weighted moments: definition and relation to parameters of several distributions expressible in inverse form". Water Resources Research. Vol. 15, Issue 5, pp. 1049-1054. October, 1979. ISSN 0043-1397.

[21] A.T. Buishand. "Extreme rainfall estimation by combining data from several sites". Journal of Hydrological Sciences. Vol. 36, Issue 6, pp. 345-365. August, 1991. ISSN: 0262-6667.

[22] P. Vauchel. "Guía de Usuario del Programa HYDRACCESS”. IRD, Programa HIBAM. 2005.

[23] J.A. Luna V. y R. Domínguez M. "Comparación de hietogramas sintéticos para diseño hidrológico". XXIV Congreso latinoamericano de hidráulica. Punta del Este, Uruguay. IAHR - AIIH. $\mathrm{N}^{\circ} 3 \mathrm{a}-144$, 13 p. 2010. 